EUROPEAN ORGANIZATION FOR NUCLEAR RESEARCH

CERN PPE/94-164

21 Oct 1994

\title{
Energy and Particle Flow in Three-Jet and Radiative Two-Jet Events from Hadronic Z Decays
}

\author{
L3 Collaboration
}

\begin{abstract}
We report on a detailed study of the energy and particle flow in the event plane of three-jet events $(q \bar{q} g)$ and radiative two-jet events $(q \bar{q} \gamma)$ in hadronic $Z$ decays recorded with the L3 detector. We find a significant decrease in particle and energy density in the angular region between quark and antiquark jets for $q \bar{q} g$ events as compared with $q \bar{q} \gamma$ events. Several QCD model predictions are compared with the observed effect.
\end{abstract}




\section{Introduction}

The measurement of energy and particle flows in the regions between jets (interjet) is known to represent an important test of QCD and fragmentation models. In three-jet events produced in $\mathrm{e}^{+} \mathrm{e}^{-}$annihilations it has been observed [1] that the region between the two quark jets $(\mathrm{q} \overline{\mathrm{q}})$ presents lower particle and energy flows relative to that which would be expected from naïve independent-fragmentation models. On the other hand, models based on string fragmentation [2] predicted this effect [3] and have been found to reproduce the data. In these models the string that generates final state particles receives a boost in the gluon direction depleting the $q \bar{q}$ region in favor of the $q g$ and $g \bar{q}$ ones. The success of these models gave origin to the name "string effect" under which the phenomenon is often known. However, it has been observed that in perturbative QCD calculations [4], coherent emission of soft gluons from the color dipoles ( $q g, g \bar{q}$ and $q \bar{q})$ produces a similar effect. Assuming "Local Parton-Hadron Duality" [5] (which is equivalent to considering the flow of final hadrons to be proportional to the flow of soft gluons), the effect should be observable at the hadron level without invoking any string fragmentation phenomenology. As a consequence a depletion is also expected from parton shower fragmentation models which include soft gluon interference effects [6].

The experimental comparison of three jet events $(q \bar{q} g)$ with two jet events having a hard photon in the final state $(q \bar{q} \gamma)$ represents a clean and model independent way of studying the "string effect" [7]. In fact, for similar kinematics the particle and energy yields in the q $\bar{q}$ region are expected to be lower for $q \bar{q} g$ than for $q \bar{q} \gamma$.

In this paper we present a comparison of the energy and particle flow distributions in the event plane of $\mathrm{q} \overline{\mathrm{q}} \mathrm{g}$ and $\mathrm{q} \overline{\mathrm{q}} \gamma$ events for similar topologies and kinematics. We use $1.5 \times 10^{6}$ hadronic events collected with the L3 detector during 1991,1992 and 1993 at $\sqrt{s} \approx 91 \mathrm{GeV}$. The results are compared with predictions from the COJETS 6.23 [8], HERWIG 5.4 [9] and JETSET 7.3 [10] Monte Carlo event generators ${ }^{1)}$. These models use a parton shower approach to describe the perturbative phase of gluon emission with differences in the treatment of "gluon coherence". The hadronization phase is described by a "string" model in JETSET and a "cluster" model in HERWIG. In COJETS partons are fragmented independently and the effects of gluon coherence are neglected.

\section{The L3 Detector}

The L3 detector [12] consists of a time expansion chamber (TEC) for tracking charged particles, a high resolution electromagnetic calorimeter of BGO crystals, a barrel of scintillation counters, a hadron calorimeter with uranium and brass absorbers and proportional wire chamber readout, and a muon spectrometer. All subdetectors are installed inside a $12 \mathrm{~m}$ diameter solenoidal magnet which provides a uniform $0.5 \mathrm{~T}$ field along the beam direction. The fiducial solid angle coverage of L3 is $99 \%$ of $4 \pi$.

The BGO energy resolution is better than $2 \%$ for electromagnetic particles above $1.5 \mathrm{GeV}$, while the angular resolution for clusters with energy above $5 \mathrm{GeV}$ is better than $0.12^{\circ}$. At 45 $\mathrm{GeV}$ the jet angular resolution is $2.5^{\circ}$ and the jet energy resolution is $10 \%$.

\footnotetext{
${ }^{1} \mathrm{~A}$ discussion of the model parameter tuning for L3 is given in reference [11].
} 


\section{Event Selection}

The selection of hadronic events is based on the energy measured in the electromagnetic and hadronic calorimeters. Events are accepted if:

$$
0.6<\frac{E_{\text {vis }}}{\sqrt{s}}<1.4, \quad \frac{\left|E_{||}\right|}{E_{\text {vis }}}<0.4, \quad \frac{E_{\perp}}{E_{\text {vis }}}<0.4, \quad N_{\text {cluster }}>12,
$$

where $E_{\text {vis }}$ is the total energy observed in the detector, $E_{\|}$is the energy imbalance along the beam direction, and $E_{\perp}$ is the transverse energy imbalance. An algorithm is used to group neighboring calorimeter signals, which are likely to be produced by the same particle, into clusters. Only clusters with a total energy above $100 \mathrm{MeV}$ are used. The number of clusters produced is proportional to the number of particles in the event, so the cut on the number of clusters, $N_{\text {cluster, }}$ rejects mainly low multiplicity non-hadronic events. Applying the same cuts to simulated events, we find that $98 \%$ of the $\mathrm{Z}$ hadronic decays are accepted. This efficiency has been found to be constant within errors for photon energies up to $45 \mathrm{GeV}$.

In the selection of $q \bar{q} g$ and $q \bar{q} \gamma$ events we pay particular attention to have similar kinematics and fiducial volumes for the two classes of events and to obtain a high purity $q \bar{q} \gamma$ sample. While jets are reconstructed in the angular region $5^{\circ}<\theta<175^{\circ}$ ( $\theta$ being the angle with respect to the LEP beam axis), photons in $q \bar{q} \gamma$ event candidates are selected only in the barrel region of the electromagnetic detector $\left(45^{\circ}<\theta<135^{\circ}\right)$. We select $q \bar{q} g$ events by applying the JADE algorithm [13] with $y_{c u t}=\mathbf{0 . 0 5}$ and E0 recombination scheme to our hadronic event sample, retaining three jet events, and then identify the gluon as the softest jet. The purity is estimated to be $(74 \pm 2) \%$ using JETSET with the Matrix Element option.

As a cross-check we perform a gluon identification by requiring the event to have a muon with momentum $p_{\mu}>4 \mathrm{GeV}$ in the second or third most energetic jet and identify the two quark jets as the most energetic jet and the one including the muon. The remaining jet is assigned to the gluon. This technique results in a higher gluon identification purity of $(85 \pm 2) \%$, but the semileptonic tag selects quark jets that include a neutrino and hence some missing energy. This makes the event kinematics different from the $q \bar{q} \gamma$ case, so we use this second method only as a cross-check.

In both cases the plane including the two quark jets is taken as the event plane and events are selected in such a way as to have the gluon jet within $10^{\circ}$ from it. Similar to the photon in $q \bar{q} \gamma$ events only gluon jets inside the central region of the detector $\left(45^{\circ}<\theta<135^{\circ}\right)$ are accepted.

The analysis faces the problems of distinguishing genuine single photons from energetic neutral hadrons and of suppressing photons emitted by the quarks at low $Q^{2}$. Events with a photon radiated at a smaller scale than a gluon should be considered as background, while events with hard photons from initial state radiation are not a background and are not removed from the sample.

In the case where the photon is emitted before any gluon radiation takes place one can make the approximation that the $q \bar{q} \gamma$ event is equivalent to a two-jet event boosted by the photon emission. If one disregards the photon, in the $q \bar{q}$ center-of-mass system the event should have the properties of a two-jet event with a total energy $\sqrt{s^{\prime}}=\sqrt{s-2 E_{\gamma} \sqrt{s}}$, where $E_{\gamma}$ is the photon energy in the laboratory frame.

Photon candidates for $q \bar{q} \gamma$ events are extracted from the three-jet sample by requiring that the least energetic jet includes a cluster of energy greater than $5 \mathrm{GeV}$ in the central region of the electromagnetic calorimeter. The requirement that this jet lies within $10^{\circ}$ of the event 
plane is tightened to $8^{\circ}$ in the $q \bar{q} \gamma$ case to take into account the better angular resolution of the photon compared to that of the gluon.

We reject most of the large background of neutral mesons decaying into photons by comparing the transverse shape of their showers to the simulated shape of a single photon [14]. The residual contamination from neutral hadrons is predicted to be $(24 \pm 2) \%$ for JETSET and $(25 \pm 2) \%$ for HERWIG. These numbers have been cross-checked by performing the shower shape analysis with a neural network which retains some discriminating power at very high energies and gives a contamination of $(26 \pm 7 \pm 6) \%$ [15].

In order to be able to compare $q \bar{q} g$ to $q \bar{q} \gamma$ events we need to reassign the hadronic activity in the photon jet to the quark jets for the $q \bar{q} \gamma$ case. To do so we recompute the jets after the photon is removed, and discard events with more than two jets.

We suppress photons radiated at a smaller scale than gluons by imposing isolation cuts. These cuts also further reduce the neutral hadron background. We first boost the event into the $q \bar{q}$ rest frame by using the photon momentum vector. Then we construct a cone of $20^{\circ}$ around the photon direction $\left(p_{x}, p_{y}, p_{z}\right)$ in a right-handed reference system $(x, y, z)$ with the $x$ axis along the direction of the most energetic jet and the $y$ axis lying in the event plane in the hemisphere opposite to the photon. We then compute the quantity

$$
E_{c}=E_{\gamma c}-E_{\gamma}-\frac{E_{c 1}+E_{c 2}+E_{c 3}}{3}
$$

where $E_{\gamma c}$ is the calorimetric energy in the cone, $E_{\gamma}$ is the photon energy, and $E_{c 1}, E_{c 2}, E_{c 3}$ are the energies in three control cones of the same aperture along the directions $\left(-p_{x},-p_{y},-p_{z}\right)$, $\left(p_{x}, p_{z}, p_{y}\right),\left(-p_{x},-p_{z},-p_{y}\right)$. The activity in any of these three cones is equivalent to that in the cone including the photon because of the event's two-jet symmetry in the boosted frame. Moreover, they never overlap since $p_{y} \gg p_{z} \approx 0$ due to the planarity of the events. The distribution of the variable $E_{c}$ is plotted in Figure 1a. Events in the region $E_{c} \simeq 0$ have hadronic activity and instrumental noise around the candidate photon similar to that which is found in symmetric regions away from jets, hence they are likely to be genuine prompt photon events. We use more than one control cone to improve the energy estimate.

A second variable $\varepsilon=E_{\gamma} / E_{\text {jet3 }}$ is defined as the ratio of the photon energy over the total energy of the original photon jet and shown in Figure $1 \mathrm{~b}$. The closer $\varepsilon$ is to 1 the more isolated is the photon in the event. After applying the cut $\left|E_{c}\right|<2 \mathrm{GeV}$ the hadronic background is reduced to about $6 \%$ of the sample and the further requirement $\varepsilon>0.8$ brings it down to $(1.8 \pm 0.6) \%$ in JETSET and to $(0.8 \pm 0.3) \%$ in HERWIG. We cross-check these background figures in a model independent way with the neural network and find a value of $(3 \pm 2 \pm 4) \%$.

In Figure 2a we show the energy flow distribution projected onto the event plane for $q \bar{q} \gamma$ JETSET events with and without the isolation cut on $\varepsilon$. We observe that the isolation cuts select events with cylindrical symmetry around the quark jets, which is consistent with the hypothesis that the only effect of the photon is a kinematic boost. Analyzing in detail the experimental data for energy flow in the region around the photon compared to the region opposite to it (Figure 2b) we see that a very tight cut on $\varepsilon$ can artificially select topologies where the region around the photon has abnormally low activity; the choice of $\varepsilon>0.8$ gives symmetric events and is therefore used.

We select $813 q \bar{q} \gamma$ events out of the full hadronic sample and use only a subset of $\sim 20000$ $\mathrm{q} \overline{\mathrm{q}} \mathrm{g}$ events which is sufficient for the statistical precision needed. Figures $3 \mathrm{a}$ and $3 \mathrm{~b}$ show the angles $A_{12}$ between quark jets and $A_{13}$ between the first jet and the photon or the gluon jet. Figure 3c compares the energy of the third-jet for the gluon and for the photon case. It is clear that a close kinematical similarity between the two classes of events has been achieved. 


\section{Results}

Particle flow plots for the $q \bar{q} \gamma$ and $q \bar{q} g$ samples are constructed by projecting, for every event, the direction of all particles onto the event plane. A particle is defined as a massless calorimetric object with an energy greater than $100 \mathrm{MeV}$. The particle flow is measured as a function of the angle increasing from jet 1 through jet 2 to jet 3 and back to jet 1 . Energy flow plots result from considering any energy deposition above $40 \mathrm{MeV}$ in the calorimeters. While in the energy flow case each event is normalized to its total visible energy, particle flow plots are not normalized since $q \bar{q} g$ events are bound to have higher multiplicity due to the gluon fragmentation. The results are shown in Figures $4 \mathrm{a}$ and $4 \mathrm{~b}$. The interquark region shows lower yield for $q \bar{q} g$ than for $q \bar{q} \gamma$ events. In order to normalize the angular distance between the two quark jets, in both the $q \bar{q} g$ and $q \bar{q} \gamma$ cases we recompute the energy and particle flows in the $q \bar{q}$ center of mass frame (Figures $4 \mathrm{c}$ and $4 \mathrm{~d}$ ). In order to be insensitive to the different energy resolution for the photon and gluon jet the new reference frame is computed using the momenta of the two quark jets.

To quantify the effect, we integrate the flow distributions of Figures $4 \mathrm{c}$ and $4 \mathrm{~d}$ in the region $\left[54^{\circ}, 135^{\circ}\right]$. This window was chosen to give maximum sensitivity to the "string effect", based on Monte Carlo studies. The individual yields integrated over the interjet window for $q \bar{q} g$ and $q \bar{q} \gamma$ are presented in Table 1 along with $R_{N}$ and $R_{E}$, the ratios of $q \bar{q} g$ to $q \bar{q} \gamma$ integrals for particle and energy flows. Also shown are the results from the same analysis applied to $2.0 \times 10^{6}$ JETSET events, $1.7 \times 10^{6}$ HERWIG events and $1.0 \times 10^{6}$ COJETS events, all fully simulated and reconstructed in the L3 detector.

The magnitude of the "string effect" is given in a model independent way by the ratio of the yields listed in the third column. We observe that $R_{E}$ and $R_{N}$ indicate a depletion of the region opposite to the gluon of $21 \%$ and $18 \%$, for the data. JETSET and HERWIG give a similar effect while COJETS shows no effect. We note that the absolute yields are underestimated by JETSET while HERWIG agrees better with the data. This illustrates the importance of measuring the "string effect" by a normalization to the $q \bar{q} \gamma$ reference sample.

\begin{tabular}{|l|c|c|c|}
\hline \hline Energy Flow & $\mathrm{q} \overline{\mathrm{q}} \boldsymbol{\gamma} \times 10^{-3}$ & $\mathrm{q} \overline{\mathrm{q}} \mathrm{g} \times 10^{-3}$ & $R_{E}$ \\
\hline Data & $8.06 \pm \mathbf{0 . 4 0}$ & $\mathbf{6 . 3 7} \pm \mathbf{0 . 0 6}$ & $\mathbf{0 . 7 9 0} \pm \mathbf{0 . 0 4 0}$ \\
JETSET 7.3 & $\mathbf{6 . 2 6} \pm \mathbf{0 . 2 9}$ & $5.39 \pm \mathbf{0 . 0 7}$ & $\mathbf{0 . 8 6 1} \pm \mathbf{0 . 0 4 2}$ \\
HERWIG 5.4 & $8.02 \pm \mathbf{0 . 4 9}$ & $\mathbf{6 . 2 9} \pm \mathbf{0 . 0 8}$ & $\mathbf{0 . 7 8 4} \pm \mathbf{0 . 0 3 9}$ \\
COJETS 6.23 & $\mathbf{6 . 7 8 \pm \mathbf { 0 . 4 7 }}$ & $7.36 \pm \mathbf{0 . 1 8}$ & $1.086 \pm \mathbf{0 . 0 7 9}$ \\
\hline \hline Particle Flow & $\mathrm{q} \overline{\mathrm{q}} \boldsymbol{\gamma}$ & $\mathrm{q} \overline{\mathrm{q} g}$ & $R_{N}$ \\
\hline Data & $1.893 \pm \mathbf{0 . 0 7 1}$ & $1.549 \pm \mathbf{0 . 0 1 1}$ & $\mathbf{0 . 8 1 8} \pm \mathbf{0 . 0 3 1}$ \\
JETSET 7.3 & $1.482 \pm \mathbf{0 . 0 4 8}$ & $1.273 \pm \mathbf{0 . 0 1 2}$ & $\mathbf{0 . 8 5 9} \pm \mathbf{0 . 0 2 9}$ \\
HERWIG 5.4 & $1.834 \pm \mathbf{0 . 0 6 6}$ & $1.441 \pm \mathbf{0 . 0 1 3}$ & $\mathbf{0 . 7 8 6} \pm \mathbf{0 . 0 2 9}$ \\
COJETS 6.23 & $1.590 \pm \mathbf{0 . 0 9 0}$ & $1.663 \pm \mathbf{0 . 0 3 0}$ & $1.046 \pm \mathbf{0 . 0 6 2}$ \\
\hline \hline
\end{tabular}

Table 1: Particle and normalized energy yields integrated from $54^{\circ}$ to $135^{\circ}$ in the q $\bar{q}$ center-ofmass frame. The third column gives the ratios $q \bar{q} g / q \bar{q} \gamma$. Energy ordering is used for the gluon jet identification. Errors are statistical. 
The following sources of systematics have been estimated:

- The subtraction of the residual neutral hadron background in the amount predicted by JETSET or HERWIG, increases the $\mathrm{R}$ values by $\Delta R_{N}=+\mathbf{0 . 0 0 5}$ and $\Delta R_{E}=+\mathbf{0 . 0 0 4}$.

- We vary the cut on $\varepsilon$ from 0.75 to 0.85 with the aim of changing the amount of photons emitted at smaller scale than gluons. We observe a change of $\Delta R_{N}= \pm 0.006$ and $\Delta R_{E}= \pm 0.010$. The systematics introduced by the $E_{c}$ cut are found to be negligible.

- The use of the DURHAM algorithm [16] with $y_{c u t}=0.02$ in the analysis, rather than the JADE algorithm, results in a $2 \%$ increase of both $R_{N}$ and $R_{E}$. This is compatible with a $5 \%$ reduction of gluon purity as predicted by JETSET. Hence we do not add this effect to the systematic error.

- For $q \bar{q} \gamma$ events, not recomputing the jet directions without the $\gamma$ candidate increases the number of events by $0.8 \%$ and increases the angle between the quark jets by $0.4^{\circ}$ on average. The resulting changes in the ratios are $\Delta R_{N}=-0.005$ and $\Delta R_{E}=-0.008$.

- The definition of a calorimetric object was modified by introducing a preclustering procedure which uses the JADE algorithm with $y_{c u t}=1.2 \times 10^{-6}$, corresponding to a mass of about $100 \mathrm{MeV}$ at LEP energies. This causes a change of $\Delta R_{N}=+0.010$ (and obviously no change in $\Delta R_{E}$ ).

- Changes of $\pm 2^{\circ}$ in the cut on the angle between the photon and the event plane produce variations $\Delta R_{N}=\Delta R_{E}= \pm 0.007$.

- The flavor composition of $q \bar{q} \gamma$ and $q \bar{q} g$ events is different because of the different quark charges resulting in different couplings to the photon. We therefore reweighted, in JETSET events, the composition of $q \bar{q} g$ events to match the flavor composition of $q \bar{q} \gamma$ ones. This was found to have no effect on $R_{N}$ or $R_{E}$.

- By a study of Monte Carlo events at generator level we have also tested the influence of cracks in the detector acceptance. The magnitude of the phenomenon is left unchanged by the addition of a blind region covering $\pm 4^{\circ}$ around the beam axis. This is the consequence of the fiducial region adopted for jet 3 in both the $q \bar{q} \gamma$ and $q \bar{q} g$ cases.

From the above study the total systematic error is \pm 0.015 for both $R_{N}$ and $R_{E}$. This gives

$$
R_{E}=0.790 \pm 0.040 \pm 0.015 \text { and } R_{N}=0.818 \pm 0.031 \pm 0.015
$$

so that the depletion of the region opposite to the gluon compared to the one opposite to the photon has a significance of $5 \sigma$ for both particle and energy flows. The results obtained by identifying the gluon jet with a $\mu$-tag give a somewhat larger effect $R_{E}=0.737 \pm 0.042 \pm 0.020$ and $R_{N}=0.753 \pm 0.032 \pm 0.020$, which is compatible with the higher gluon purity.

It has been remarked [17] that the observed effect could have a purely kinematic origin, being caused by the difference between the massless photon and the effective mass of the gluon jet. In this scenario the quark jets of the $q \bar{q} g$ events, having less energy to share, are slimmer and result in lower interjet activity. In fact, we observe a small difference between the $q \bar{q} \gamma$ and $q \bar{q} g$ kinematics as a shift of the order of $10 \%$ in the masses of the two quark jets in our data and in all the Monte Carlo models used. The difference also occurs for COJETS even though it does not reproduce the "string effect". Also, this mass shift is reduced by half if the jets are 
not recomputed after the removal of the photon, while the magnitude of the "string effect" is left unchanged. We conclude that the effect cannot be explained on these grounds.

As noted by several authors $[4,18]$ the magnitude of the "string effect" is expected to increase by selecting for each event only particles with a large momentum component, $P_{\text {out }}$, perpendicular to the event plane. This phenomenon, observed by MarkII [19] and JADE [20] at lower energies, is predicted by perturbative QCD to decrease at LEP energy and to vanish asymptotically. In practice the investigation of the $P_{\text {out }}$ dependence is difficult as the $P_{\text {out }}$ selection reduces further the already limited statistical power of the $q \bar{q} \gamma$ control sample. To partially overcome this problem we use here the cylindrical symmetry of the $q \bar{q} \gamma$ event boosted to the $q \bar{q}$ rest frame. Because of this symmetry the definition of the event plane is arbitrary in the case of $q \bar{q} \gamma$ events and, instead of selecting one plane for the flow calculations, we can average the distributions obtained from all the possible planes containing the jet axis. This means that a particle gives a contribution to a specific flow-plot bin which is a function of its angle $\alpha$ relative to the $q \bar{q}$ axis. In the case of a cut on $P_{\text {out }}$ the condition $P_{\text {out }}>P_{\text {out }}^{\text {cut }}$ is applied in each plane separately. The systematic effect introduced by the above algorithm [15] has been found to be negligible by a study of JETSET at generator level. In the case of $q \bar{q} g$ events where the event plane has a precise meaning even in the $q \bar{q}$ rest frame (and the statistics is more abundant) we select particles having $P_{\text {out }}>0.2$ or $0.3 \mathrm{GeV}$. We then compare the region $\left[0^{\circ}, 180^{\circ}\right]$ of the $q \bar{q} g$ flow plot with that obtained for $q \bar{q} \gamma$. The bin-by-bin ratio of $q \bar{q} g$ to $q \bar{q} \gamma$ events is shown in Figures 5a and 5b for energy and particle flows, with no $P_{\text {out }}$ cut. The observed dip corresponds to the "string effect". In Figures 5c and 5d we plot the variation of the effect when a cut $P_{\text {out }}>0.2 \mathrm{GeV}$ is applied.

The double ratios $\rho_{E}\left(P_{\text {out }}\right)=R_{E}^{P_{\text {out }}} / R_{E}$ and $\rho_{N}\left(P_{\text {out }}\right)=R_{N}^{P_{\text {out }}} / R_{N}$ for $P_{\text {out }}>0.2 \mathrm{GeV}$ and $P_{\text {out }}>0.3 \mathrm{GeV}$ are shown in Table 2 . We only give statistical errors since the systematics in the double ratios cancel and are found to be negligible. In a similar fashion detector corrections are also negligible for the quantities $\rho_{E}$ and $\rho_{N}$ so that high statistics generator level runs are used for JETSET, HERWIG and COJETS in the table.

\begin{tabular}{|l|c|c|c|c|}
\hline \hline & $\rho_{E}(\mathbf{0 . 2 \mathrm { GeV } )}$ & $\rho_{N}(\mathbf{0 . 2 \mathrm { GeV } )}$ & $\rho_{E}(\mathbf{0 . 3 \mathrm { GeV } )}$ & $\rho_{N}(\mathbf{0 . 3 \mathrm { GeV } )}$ \\
\hline Data & $\mathbf{0 . 9 8 9} \pm \mathbf{0 . 0 2 8}$ & $\mathbf{0 . 9 1 1} \pm \mathbf{0 . 0 3 6}$ & $1.002 \pm \mathbf{0 . 0 3 8}$ & $\mathbf{0 . 9 0 8} \pm \mathbf{0 . 0 5 2}$ \\
JETSET 7.3 & $\mathbf{0 . 9 8 2} \pm \mathbf{0 . 0 1 3}$ & $\mathbf{0 . 9 0 0} \pm \mathbf{0 . 0 1 4}$ & $\mathbf{0 . 9 8 4} \pm \mathbf{0 . 0 1 7}$ & $\mathbf{0 . 8 7 3} \pm \mathbf{0 . 0 2 0}$ \\
HERWIG 5.4 & $1.007 \pm \mathbf{0 . 0 1 4}$ & $\mathbf{0 . 9 4 0} \pm \mathbf{0 . 0 1 7}$ & $1.028 \pm \mathbf{0 . 0 1 9}$ & $\mathbf{0 . 9 4 3} \pm \mathbf{0 . 0 2 4}$ \\
COJETS 6.23 & $1.037 \pm \mathbf{0 . 0 1 7}$ & $1.009 \pm \mathbf{0 . 0 2 2}$ & $1.057 \pm \mathbf{0 . 0 2 4}$ & $1.017 \pm \mathbf{0 . 0 3 0}$ \\
\hline \hline
\end{tabular}

Table 2: Double ratios $\rho_{E}\left(P_{\text {out }}\right)=R_{E}^{P_{\text {out }}} / R_{E}$ and $\rho_{N}\left(P_{\text {out }}\right)=R_{N}^{P_{\text {out }}} / R_{N}$ computed over the interval $\left[54^{\circ}, 135^{\circ}\right]$. Data is compared to Monte Carlo generators for energy and particle flows. The systematics give negligible contribution to the errors.

Within the present statistics the particle flow shows an enhancement of the "string effect" at large $P_{\text {out }}$ with a $\sim 3 \sigma$ significance, while the energy flow shows no enhancement. Both JETSET and HERWIG follow the data while the comparison with the MarkII and JADE lower energy measurements gives a picture consistent with a vanishing dependence on $P_{\text {out }}$ at large center of mass energies. This is compatible with perturbative QCD predictions. 


\section{Conclusions}

We have studied the energy and particle flow in the region opposite to the gluon jet in three-jet events by comparing them with kinematically analogous events with two jets and one hard isolated photon. We find clear evidence, with a significance of 5 standard deviations, for lower flows in the three-jet event case as predicted by the string fragmentation model and by soft gluon coherence in the context of perturbative QCD. This effect is correctly reproduced by the JETSET and HERWIG event generators. We find, however, that the COJETS event generator does not reproduce the data. We have extended the analysis to particles having a large momentum component outside the event plane and found a small enhancement only in the case of particle flow.

\section{Acknowledgments}

We express our gratitude to the CERN accelerator divisions for the excellent performance of the LEP machine. We acknowledge the effort of all engineers and technicians who have participated in the construction and maintenance of this experiment.

We wish to thank T. Sjöstrand for many helpful discussions. 


\section{The L3 Collaboration:}

M.Acciarri ${ }^{26}$ A.Adam, ${ }^{43}$ O.Adriani ${ }^{16}$ M.Aguilar-Benitez ${ }^{25}$ S.Ahlen, ${ }^{10}$ J.Alcaraz ${ }^{25}$ A.Aloisio, ${ }^{28}$ G.Alverson, ${ }^{11}$ M.G.Alviggi ${ }^{28}$ G.Ambrosi, ${ }^{33}$ Q.An ${ }^{18}$ H.Anderhub ${ }^{46}$ A.L.Anderson ${ }^{15}$ V.P.Andreev ${ }^{37}$ T.Angelescu, ${ }^{12}$ L.Antonov $^{40}$ D.Antreasyan, G.Alkhazov, ${ }^{37}$ P.Arce, ${ }^{25}$ A.Arefiev ${ }^{27}$ T.Azemoon, T.Aziz, P.V.K.S.Baba, ${ }^{18}$ P.Bagnaia, ${ }^{36,17}$ J.A.Bakken, ${ }^{35}$ L.Baksay, ${ }^{42}$ R.C.Ball, ${ }^{3}$ S.Banerjee, K.Banicz ${ }^{43}$ R.Barillère ${ }^{17}$ L.Barone ${ }^{36}$ A.Baschirotto, ${ }^{26}$ M.Basile ${ }^{8}$ R.Battiston, ${ }^{33}$ A.Bay, ${ }^{22}$ F.Becattini ${ }^{16}$ U.Becker, ${ }^{15}$ F.Behner, ${ }^{46}$ Gy.L.Bencze, ${ }^{13}$ J.Berdugo, ${ }^{25}$ P.Berges, ${ }^{15}$ B.Bertucci ${ }^{17}$ B.L.Betev, ${ }^{40,46}$ M.Biasini, ${ }^{33}$ A.Biland, ${ }^{46}$ G.M.Bilei ${ }^{33}$ R.Bizzarri, ${ }^{36}$ J.J.Blaising, G.J.Bobbink, ${ }^{172}$ R.Bock, A.Böhm, B.Borgia, ${ }^{36}$ A.Boucham, D.Bourilkov, ${ }^{46}$ M.Bourquin, ${ }^{19}$ D.Boutigny, ${ }^{17}$ B.Bouwens, E.Brambilla, ${ }^{15}$ J.G.Branson, ${ }^{38}$ V.Brigljevic, ${ }^{46}$ I.C.Brock, ${ }^{34}$ M.Brooks ${ }^{23}$ A.Bujak, ${ }^{43}$ J.D.Burger, ${ }^{15}$ W.J.Burger ${ }^{19}$ C.Burgos ${ }^{25}$ J.Busenitz, ${ }^{42}$ A.Buytenhuijs, ${ }^{30}$ A.Bykov ${ }^{37}$ X.D.Cai ${ }^{18}$ M.Capell ${ }^{15}$ G.Cara Romeo, M.Caria, ${ }^{83}$ G.Carlino, ${ }^{28}$ A.M.Cartacci ${ }^{16}$ J.Casaus, ${ }^{25}$ R.Castello, ${ }^{26}$ N.Cavallo, ${ }^{28}$ M.Cerrada, ${ }^{25}$ F.Cesaroni ${ }^{36}$ M.Chamizo, ${ }^{25}$ Y.H.Chang, ${ }^{48}$ U.K.Chaturvedi, ${ }^{18}$ M.Chemarin, ${ }^{24}$ A.Chen, ${ }^{48}$ C.Chen, ${ }^{6}$ G.Chen, ${ }^{6,46}$ G.M.Chen, H.F.Chen ${ }^{20}$ H.S.Chen, M.Chen, ${ }^{6}$ G.Chiefari ${ }^{28}$ C.Y.Chien ${ }^{5}$ M.T.Choi ${ }^{41}$ S.Chung, ${ }^{15}$ L.Cifarelli, ${ }^{8}$ F.Cindolo, ${ }^{8}$ C.Civinini ${ }^{16}{ }^{16}$.Clare, ${ }^{15}$ R.Clare, ${ }^{15}$ T.E.Coan, ${ }^{23}$ H.O.Cohn, ${ }^{31}$ G.Coignet, N.Colino, ${ }^{4}$ S.Costantini ${ }^{36}$ F.Cotorobai ${ }^{12}$ B.de la Cruz ${ }^{25}$ X.T.Cui, ${ }^{18}$ X.Y.Cui ${ }^{18}$ T.S.Dai ${ }^{15}$ R.D'Alessandro, ${ }^{16}$ R.de Asmundis, ${ }^{28}$ A.Degré, K.Deiters, ${ }^{44}$ E.Dénes, ${ }^{13}$ P.Denes, ${ }^{35}$ F.DeNotaristefani, ${ }^{36}$ D.DiBitonto, ${ }^{42}$ M.Diemoz ${ }^{36}$ H.R.Dimitrov ${ }^{40}$ C.Dionisi ${ }^{36}$ M.Dittmar, ${ }^{46}$ L.Djambazov ${ }^{46}$ I.Dorne, M.T.Dova ${ }^{48, \sharp}$ E.Drago ${ }^{28}$ D.Duchesneau, ${ }^{17}$ F.Duhem, ${ }^{4}$ P.Duinker, ${ }^{2}$ I.Duran, ${ }^{39}$ S.Dutta, ${ }^{9}$ S.Easo, ${ }^{33}$ H.El Mamouni, ${ }^{24}$ A.Engler, ${ }^{34}$ F.J.Eppling, ${ }^{15}$ F.C.Erné, ${ }^{27}$ P.Extermann, ${ }^{19}$ R.Fabbretti ${ }^{44}$ M.Fabre ${ }^{44}$ S.Falciano ${ }^{36}$ A.Favara ${ }^{16}$ J.Fay ${ }^{24}$ M.Felcini ${ }^{46}$ T.Ferguson, ${ }^{34}$ D.Fernandez ${ }^{25}$ G.Fernandez ${ }^{25}$ F.Ferroni ${ }^{36}$ H.Fesefeldt, ${ }^{1}$ E.Fiandrini ${ }^{33}$ J.H.Field, ${ }^{19}$ F.Filthaut, ${ }^{30}$ P.H.Fisher, ${ }^{5}$ G.Forconi ${ }^{15}$ L.Fredj ${ }^{19}$ K.Freudenreich ${ }^{46}$ M.Gailloud ${ }^{22}{ }^{\text {Yu.Galaktionov }}{ }^{27,15}$ E.Gallo, ${ }^{16}$ S.N.Ganguli, P.Garcia-Abia, ${ }^{25}$ S.Gentile, ${ }^{36}$ J.Gerald, N.Gheordanescu, ${ }^{12}$ S.Giagu, ${ }^{36}$ S.Goldfarb, ${ }^{22}$ J.Goldstein, ${ }^{10}$ Z.F.Gong, E.Gonzalez ${ }^{25}$ A.Gougas, D.Goujon, ${ }^{19}$ G.Gratta, ${ }^{32}$ M.W.Gruenewald, C.Gu, ${ }^{18}$ M.Guanziroli, ${ }^{18}$ V.K.Gupta, ${ }^{35}$ A.Gurtu, H.R.Gustafson, L.J.Gutay, ${ }^{43}$ A.Hasan, ${ }^{29}$ D.Hauschildt, ${ }^{2}$ J.T.He, ${ }^{6}$ T.Hebbeker, M.Hebert ${ }^{38}$ A.Hervé ${ }^{17}$ K.Hilgers, H.Hofer, ${ }^{46}$ H.Hoorani ${ }^{19}$ S.R.Hou ${ }^{48}$ G.Hu${ }^{18}$ B.Ille, ${ }^{24}$ M.M.Ilyas ${ }^{18}$ V.Innocente, ${ }^{17}$ H.Janssen, B.N.Jin, L.W.Jones, P.de Jong, ${ }^{15}$ I.Josa-Mutuberria, ${ }^{17}$ A.Kasser, ${ }^{22}$ R.A.Khan ${ }^{18}$ Yu.Kamyshkov ${ }^{31}$ P.Kapinos, ${ }^{45}$ J.S.Kapustinsky ${ }^{23}$ Y.Karyotakis, ${ }^{17}$ M.Kaur ${ }^{18}$ S.Khokhar ${ }^{18}$ M.N.Kienzle-Focacci ${ }^{19}$ D.Kim, ${ }^{5}$ J.K.Kim, ${ }^{41}$ S.C.Kim, ${ }^{41}$ Y.G.Kim, ${ }^{41}$ W.W.Kinnison, ${ }^{23}$ A.Kirkby, ${ }^{32}$ D.Kirkby ${ }^{32}$ J.Kirkby, ${ }^{17}$ S.Kirsch, ${ }^{45}$ W.Kittel ${ }^{30}$ A.Klimentov ${ }^{15,27}$ A.C.König, ${ }^{30}$ E.Koffeman, O.Kornadt, ${ }^{1}$ V.Koutsenko, ${ }^{1,27}$ A.Koulbardis, ${ }^{37}$ R.W.Kraemer, ${ }^{34}$ T.Kramer, ${ }^{15}$ V.R.Krastev, ${ }^{40,33}$ W.Krenz, H.Kuijten, ${ }^{30}$ K.S.Kumar, ${ }^{14}$ A.Kunin, ${ }^{15,27}$ P.Ladron de Guevara, ${ }^{25,17}$ G.Landi ${ }^{16}$ D.Lanske, S.Lanzano, ${ }^{28}$ P.Laurikainen, ${ }^{21}$ A.Lebedev ${ }^{15}$ P.Lebrun, ${ }^{24}$ P.Lecomte ${ }^{46}$ P.Lecoq, ${ }^{17}$ P.Le Coultre, ${ }^{46}$ D.M.Lee, ${ }^{23}$ J.S.Lee, ${ }^{41}$ K.Y.Lee ${ }^{41}$ I.Leedom, ${ }^{11}$ C.Leggett, ${ }^{3}$ J.M.Le Goff, ${ }^{17}$ R.Leiste ${ }^{45}$ M.Lenti, ${ }^{16}$ E.Leonardi ${ }^{36}$ P.Levtchenko, ${ }^{37}$ C.Li ${ }^{20,18}$ E.Lieb, ${ }^{45}$ W.T.Lin ${ }^{48}$ F.L.Linde, B.Lindemann, ${ }^{1}$ L.Lista ${ }^{28}{ }^{2}$ Y.Liu ${ }^{18}$ W.Lohmann, ${ }^{45}$ E.Longo, ${ }^{36}$ W.Lu ${ }^{32}$ Y.S.Lu, ${ }^{6}$ J.M.Lubbers, ${ }^{17}$ K.Lübelsmeyer, C.Luci ${ }^{36}$ D.Luckey ${ }^{15}{ }^{15}$.Ludovici ${ }^{36}$ L.Luminari $^{36}$ W.Lustermann, ${ }^{44}$ W.G.Ma ${ }^{20}$ M.MacDermott ${ }^{46}$ M.Maity ${ }^{9}$ L.Malgeri ${ }^{36}$ R.Malik ${ }^{18}$ A.Malinin, ${ }^{27}$ C.Maña ${ }^{25}$ S.Mangla, M.Maolinbay, ${ }^{46}$ P.Marchesini, ${ }^{46}$ A.Marin, ${ }^{10}$ J.P.Martin ${ }^{24}$ F.Marzano, ${ }^{36}$ G.G.G.Massaro, K.Mazumdar, P.McBride ${ }^{14}$ T.McMahon, ${ }^{43}$ D.McNally ${ }^{38}$ S.Mele ${ }^{28}$ M.Merk ${ }^{34}$ L.Merola ${ }^{28}$ M.Meschini ${ }^{16}$ W.J.Metzger ${ }^{30}$ Y.Mi $^{22}$ A.Mihul, ${ }^{12}$ G.B.Mills, ${ }^{23}$ Y.Mir, ${ }^{18}$ G.Mirabelli, ${ }^{36}$ J.Mnich, M.Möller, V.Monaco, ${ }^{16}$ B.Monteleoni ${ }^{16}$ R.Morand, S.Morganti ${ }^{36}$ N.E.Moulai, ${ }^{18}$ R.Mount, ${ }^{32}$ S.Müller ${ }^{1}$ E.Nagy ${ }^{13}$ M.Napolitano, ${ }^{28}$ F.Nessi-Tedaldi, ${ }^{46}$ H.Newman, ${ }^{32}$ M.A.Niaz ${ }^{18}$ A.Nippe, H.Nowak ${ }^{45}$ G.Organtini ${ }^{36}$ R.Ostonen, ${ }^{21}$ D.Pandoulas, ${ }^{1}$ S.Paoletti ${ }^{36}$ P.Paolucci ${ }^{28}$ G.Pascale ${ }^{36}$ G.Passaleva, ${ }^{16,33}$ S.Patricelli, ${ }^{28}$ T.Paul, M.Pauluzzi, ${ }^{33}$ C.Paus, F.Pauss ${ }^{46}{ }^{16}$ Y.J.Pei, ${ }^{1}$ S.Pensotti ${ }^{26}$ D.Perret-Gallix ${ }^{4}$ A.Pevsner, D.Piccolo, ${ }^{28}$ M.Pieri, ${ }^{16}$ J.C.Pinto, ${ }^{34}$ P.A.Piroué, ${ }^{35}$ E.Pistolesi ${ }^{16}$ F.Plasil, ${ }^{31}$ V.Plyaskin, ${ }^{27}$ M.Pohl ${ }^{46}$ V.Pojidaev, ${ }^{276}$ H.Postema, ${ }^{15}$ N.Produit, ${ }^{19}$ J.M.Qian, K.N.Qureshi, ${ }^{18}$ R.Raghavan, G.Rahal-Callot, ${ }^{46}$ P.G.Rancoita, ${ }^{26}$ M.Rattaggi, ${ }^{26}$ G.Raven, P.Razis, ${ }^{29}$ K.Read, ${ }^{31}$ M.Redaelli, ${ }^{26}$ D.Ren, ${ }^{46}$ Z.Ren $^{18}$ M.Rescigno, ${ }^{36}$ S.Reucroft, ${ }^{11}$ A.Ricker, S.Riemann, ${ }^{45}$ B.C.Riemers, ${ }^{43}$ K.Riles, O.Rind, H.A.Rizvi, ${ }^{3}{ }^{3}$ S.Ro ${ }^{41}$ A.Robohm, ${ }^{46}$ F.J.Rodriguez ${ }^{25}$ B.P.Roe, M.Röhner, ${ }^{3}$ S.Röhner ${ }^{1}$ L.Romero, ${ }^{25}$ S.Rosier-Lees, R.Rosmalen, ${ }^{30}$ Ph.Rosselet ${ }^{22}$ W.van Rossum, S.Roth, A.Rubbia, ${ }^{15}$ J.A.Rubio, ${ }^{17}$ H.Rykaczewski, ${ }^{46}$ J.Salicio, ${ }^{17}$ J.M.Salicio, ${ }^{25}$ E.Sanchez ${ }^{25}$ G.S.Sanders, ${ }^{23}$ A.Santocchia, ${ }^{33}$ M.E.Sarakinos, ${ }^{43}$ S.Sarkar, G.Sartorelli, ${ }^{18}$ M.Sassowsky, G.Sauvage, C.Schäfer, V.Schegelsky ${ }^{37}$ D.Schmitz, P.Schmitz, M.Schneegans, ${ }^{4}$ N.Scholz ${ }^{46}$ H.Schopper ${ }^{47}$ D.J.Schotanus ${ }^{30}$ S.Shotkin ${ }^{15}$ H.J.Schreiber, ${ }^{45}$ J.Shukla, ${ }^{34}$ R.Schulte, K.Schultze, J.Schwenke, G.Schwering, C.Sciacca ${ }^{18}{ }^{1}$ I.Scott ${ }^{14}$ R.Sehgal ${ }^{18}$ P.G.Seiler, ${ }^{44}$ J.C.Sens, ${ }^{17}{ }^{2}$ L.Servoli, ${ }^{33}$ I.Sheer, ${ }^{38}$ S.Shevchenko, ${ }^{32}$ X.R.Shi ${ }^{32}$ E.Shumilov ${ }^{27}$ V.Shoutko, ${ }^{27}$ D.Son, A.Sopczak, ${ }^{17}$ V.Soulimov, ${ }^{28}$ C.Spartiotis, ${ }^{21}$ T.Spickermann, 1 P.Spillantini, ${ }^{16}$ M.Steuer, ${ }^{15}$ D.P.Stickland, ${ }^{35}$ F.Sticozzi ${ }^{15}$ H.Stone, ${ }^{35}$ K.Strauch, ${ }^{14}$ K.Sudhakar, G.Sultanov, ${ }^{18}$ L.Z.Sun, ${ }^{20,18}$ G.F.Susinno, ${ }^{19}$ H.Suter, ${ }^{46}$ J.D.Swain, ${ }^{18}$ A.A.Syed, X.W.Tang, L.Taylor, ${ }^{11}$ R.Timellini, ${ }^{8}$ Samuel C.C.Ting, ${ }^{15}$ S.M.Ting, ${ }^{15}$ O.Toker, ${ }^{33}$ M.Tonutti, S.C.Tonwar, J.Tóth, ${ }^{13}$ G.Trowitzsch, ${ }^{45}$ A.Tsaregorodtsev ${ }^{37}$ G.Tsipolitis, ${ }^{34}$ C.Tully ${ }^{35}$ J.Ulbricht ${ }^{46}$ L.Urbán, ${ }^{13}$ U.Uwer, E.Valente, ${ }^{36}$ R.T.Van de Walle, ${ }^{30}$ I.Vetlitsky, ${ }^{27}$ G.Viertel, ${ }^{46}$ P.Vikas, ${ }^{18}$ U.Vikas, ${ }^{18}$ M.Vivargent, ${ }^{4}$ H.Vogel ${ }^{34}$ H.Vogt ${ }^{45}$ I.Vorobiev, ${ }^{14,27}$ A.A.Vorobyov ${ }^{37}$ An.A.Vorobyov, ${ }^{37}$ L.Vuilleumier ${ }^{22}$ M.Wadhwa, ${ }^{25}$ W.Wallraff, ${ }^{1}$ J.C.Wang, ${ }^{15}$ X.L.Wang, ${ }^{20}$ Y.F.Wang, ${ }^{15}$ Z.M.Wang, ${ }^{18,20}$ A.Weber, J.Weber, ${ }^{46}$ R.Weill, ${ }^{22}$ C.Willmott ${ }^{25}$ F.Wittgenstein, ${ }^{17}$ D.Wright ${ }^{35}$ S.X.Wu, ${ }^{18}$ S.Wynhoff, Z.Z.Xu ${ }^{20}$ B.Z.Yang, ${ }^{20}$ C.G.Yang, ${ }^{6}$ G.Yang ${ }^{18}$ X.Y.Yao ${ }^{6}$ C.H.Ye, ${ }^{18}$ J.B.Ye, ${ }^{20}$ Q.Ye, S.C.Yeh, ${ }^{48}$ J.M.You, ${ }^{18}$ N.Yunus, ${ }^{18}$ M.Yzerman, C.Zaccardelli, ${ }^{32}$ P.Zemp, ${ }^{46}$ M.Zeng, ${ }^{18}$ Y.Zeng, D.H.Zhang, Z.P.Zhang, ${ }^{20,18}$ B.Zhou, ${ }^{10}$ G.J.Zhou, J.F.Zhou, R.Y.Zhu, ${ }^{62}$ A.Zichichi, ${ }^{8,18}$ B.C.C.van der Zwaan. 
1 I. Physikalisches Institut, RWTH, D-52056 Aachen, FRG ${ }^{\S}$

III. Physikalisches Institut, RWTH, D-52056 Aachen, FRG ${ }^{\S}$

2 National Institute for High Energy Physics, NIKHEF, NL-1009 DB Amsterdam, The Netherlands

3 University of Michigan, Ann Arbor, MI 48109, USA

4 Laboratoire d'Annecy-le-Vieux de Physique des Particules, LAPP,IN2P3-CNRS, BP 110, F-74941

Annecy-le-Vieux CEDEX, France

5 Johns Hopkins University, Baltimore, MD 21218, USA

6 Institute of High Energy Physics, IHEP, 100039 Beijing, China

7 Humboldt University, D-10099 Berlin, FRG

8 INFN-Sezione di Bologna, I-40126 Bologna, Italy

9 Tata Institute of Fundamental Research, Bombay 400 005, India

10 Boston University, Boston, MA 02215, USA

11 Northeastern University, Boston, MA 02115, USA

12 Institute of Atomic Physics and University of Bucharest, R-76900 Bucharest, Romania

13 Central Research Institute for Physics of the Hungarian Academy of Sciences, H-1525 Budapest 114, Hungary

14 Harvard University, Cambridge, MA 02139, USA

15 Massachusetts Institute of Technology, Cambridge, MA 02139, USA

16 INFN Sezione di Firenze and University of Florence, I-50125 Florence, Italy

17 European Laboratory for Particle Physics, CERN, CH-1211 Geneva 23, Switzerland

18 World Laboratory, FBLJA Project, CH-1211 Geneva 23, Switzerland

19 University of Geneva, CH-1211 Geneva 4, Switzerland

20 Chinese University of Science and Technology, USTC, Hefei, Anhui 230 029, China

21 SEFT, Research Institute for High Energy Physics, P.O. Box 9, SF-00014 Helsinki, Finland

22 University of Lausanne, CH-1015 Lausanne, Switzerland

23 Los Alamos National Laboratory, Los Alamos, NM 87544, USA

24 Institut de Physique Nucléaire de Lyon, IN2P3-CNRS,Université Claude Bernard, F-69622 Villeurbanne Cedex, France

25 Centro de Investigaciones Energeticas, Medioambientales y Tecnologicas, CIEMAT, E-28040 Madrid, Spain

26 INFN-Sezione di Milano, I-20133 Milan, Italy

27 Institute of Theoretical and Experimental Physics, ITEP, Moscow, Russia

28 INFN-Sezione di Napoli and University of Naples, I-80125 Naples, Italy

29 Department of Natural Sciences, University of Cyprus, Nicosia, Cyprus

30 University of Nymegen and NIKHEF, NL-6525 ED Nymegen, The Netherlands

31 Oak Ridge National Laboratory, Oak Ridge, TN 37831, USA

32 California Institute of Technology, Pasadena, CA 91125, USA

33 INFN-Sezione di Perugia and Universitá Degli Studi di Perugia, I-06100 Perugia, Italy

34 Carnegie Mellon University, Pittsburgh, PA 15213, USA

35 Princeton University, Princeton, NJ 08544, USA

36 INFN-Sezione di Roma and University of Rome, "La Sapienza", I-00185 Rome, Italy

37 Nuclear Physics Institute, St. Petersburg, Russia

38 University of California, San Diego, CA 92093, USA

39 Dept. de Fisica de Particulas Elementales, Univ. de Santiago, E-15706 Santiago de Compostela, Spain

40 Bulgarian Academy of Sciences, Institute of Mechatronics, BU-1113 Sofia, Bulgaria

41 Center for High Energy Physics, Korea Advanced Inst. of Sciences and Technology, 305-701 Taejon, Republic of Korea

42 University of Alabama, Tuscaloosa, AL 35486, USA

43 Purdue University, West Lafayette, IN 47907, USA

44 Paul Scherrer Institut, PSI, CH-5232 Villigen, Switzerland

45 DESY-Institut für Hochenergiephysik, D-15738 Zeuthen, FRG

46 Eidgenössische Technische Hochschule, ETH Zürich, CH-8093 Zürich, Switzerland

47 University of Hamburg, 22761 Hamburg, FRG

48 High Energy Physics Group, Taiwan, China

$\S$ Supported by the German Bundesministerium für Forschung und Technologie

$\doteqdot$ Supported by the Hungarian OTKA fund under contract number 2970 .

\# Also supported by CONICET and Universidad Nacional de La Plata, CC 67, 1900 La Plata, Argentina

$\dagger$ Deceased. 


\section{References}

[1] JADE Collaboration, W. Bartel et al., Phys. Lett. B101 (1981) 129-134;

JADE Collaboration, W. Bartel et al., Z. Phys. C21 (1983) 37-52;

JADE Collaboration, W. Bartel et al., Phys. Lett. B134 (1984) 275-280;

TASSO Collaboration, M. Althoff et al., Z. Phys. C29 (1985) 29-40;

TPC collaboration, H. Aihara et al., Z. Phys. C28 (1985) 31-44;

OPAL Collaboration, P.D. Acton et al., Z. Phys. C58 (1993) 387-404;

OPAL Collaboration, M.Z. Akrawy et al., Phys. Lett. B261 (1991) 334-346.

[2] B. Andersson, G. Gustafson, G. Ingelman and T. Sjöstrand, Phys. Rep. 97 (1983) 31.

[3] B. Andersson, G. Gustafson and T. Sjöstrand, Phys. Lett. B94 (1980) 211.

[4] Y.I. Azimov, Y.L. Dokshitzer, V.A. Khoze, S.I. Troyan, Phys. Lett. B165 (1985) 147-150;

Y.I. Azimov, Y.L. Dokshitzer, V.A. Khoze, S.I. Troyan, Yad. Fiz. 43 (1986) 159;

Y.L. Dokshitzer, V.A. Khoze, A.H. Mueller, S.I. Troyan, Basics of Perturbative QCD éd. Frontières (1991).

[5] Y.L. Dokshitzer, S.I. Troyan, Proceedings of the XIX Winter School of the LNPI Vol. 1 (1984) 144;

Y.I. Azimov, Y.L. Dokshitzer, V.A. Khoze, S.I. Troyan, Z. Phys. C27 (1985) 65-72.

[6] G. Marchesini, B. Webber, Nucl. Phys. B238 (1981) 1-29.

[7] TPC $/ 2 \gamma$ collaboration, H. Aihara et al., Phys. Rev. Lett. 57 (1986) 945-948;

MARK II Collaboration, P.D. Seldon et al., Phys. Rev. Lett. 57 (1986) 1398-1401;

JADE Collaboration, F. Ould Saada et al., Z. Phys. C39 (1988) 1-6.

[8] R. Odorico, Comput. Phys. Commun. 32 (1984) 139-172;

R. Odorico, Comput. Phys. Commun. 34 (1985) 431-436 (erratum).

[9] G. Marchesini, B. Webber, Nucl. Phys. B310 (1988) 461-526;

I.G. Knowles, Nucl. Phys. B310 (1988) 571-588.

[10] T. Sjöstrand, Comput. Phys. Commun. 39 (1986) 347-407;

T. Sjöstrand, M. Bengtsson, Comput. Phys. Commun. 43 (1987) 367-379.

[11] L3 Collaboration, B. Adeva et al., Z. Phys. C55 (1992) 39-61.

[12] L3 Collaboration, B. Adeva et al., Nucl. Inst. Meth. A289 (1990) 35-102.

[13] JADE Collaboration, W. Bartel et al., Z. Phys. C33 (1986) 23-31;

JADE Collaboration, S. Bethke et al., Phys. Lett. B213 (1988) 235-241.

[14] L3 Collaboration, O. Adriani et al., Phys. Lett. B292 (1992) 472-484.

[15] D. Duchesneau, PhD thesis \#2644, University of Geneva (1993) unpublished.

[16] S. Catani et al., Phys. Lett. B269 (1991) 432;

N. Brown and W.J. Stirling, Z. Phys. C53 (1992) 629.

[17] G. Ballocchi and R. Odorico, Nucl. Phys. B345 (1990) 173-185.

[18] V. Khoze, L. Lönnblad, Phys. Lett. B241 (1990) 123.

[19] MARK II Collaboration, P.D. Seldon et al., Phys. Rev. Lett. 57 (1986) 1398-1401.

[20] JADE Collaboration, F. Ould Saada et al., Z. Phys. C39 (1988) 1-6. 


\section{List of Figures}

1 Isolation variables (a) $E_{c}$, (b) $\varepsilon$ after the cut $\left|E_{c}\right|<2 \mathrm{GeV}$ has been applied. Solid points represent the data, while the histogram represents the JETSET prediction. The background contribution from neutral hadrons is shown as the hatched area. The arrows represent the cuts used.

2 (a) Energy distribution projected onto the event plane in the $q \bar{q}$ rest frame for JETSET $q \bar{q} \gamma$ events after removal of the photon, which otherwise appears around $260^{\circ}$. Angles run from highest energetic jet direction towards the second jet. Neutral hadron background is removed and the $E_{c}$ cut has been applied. (b) Relative energy flow difference $\delta$ between the photon region $\left[234^{\circ}, 297^{\circ}\right]$ and the symmetric one $\left[63^{\circ}, 126^{\circ}\right]$ for data as a function of the cut on $\varepsilon$.

3 (a) Angle $A_{12}$ between the two quark jets and (b) between the most energetic jet and the third jet $A_{13}$. (c) Energy of the third jet $E_{3}$.

4 (a) Distribution of the normalized energy flow and (b) particle flow in the laboratory frame. (c) and (d) are the corresponding distributions in the $q \bar{q}$ center of mass frame, after the photon has been removed. The arrows show the angular range used to measure the effect.

5 Bin-by-bin ratios of the $q \bar{q} g$ and $q \bar{q} \gamma$ (a) energy and (b) particle flow plots after the application of the algorithm described in the text to $q \bar{q} \gamma$ events. The theoretical predictions have statistical uncertainties of similar magnitude to the ones shown for data. (c) and (d) show the ratios of the distributions with and without a $0.2 \mathrm{GeV} P_{\text {out }}$ cut. Systematic errors are not shown in (a) and (b), while they are negligible in $(c)$ and $(d)$. The arrows show the angular range used to measure the effect. 

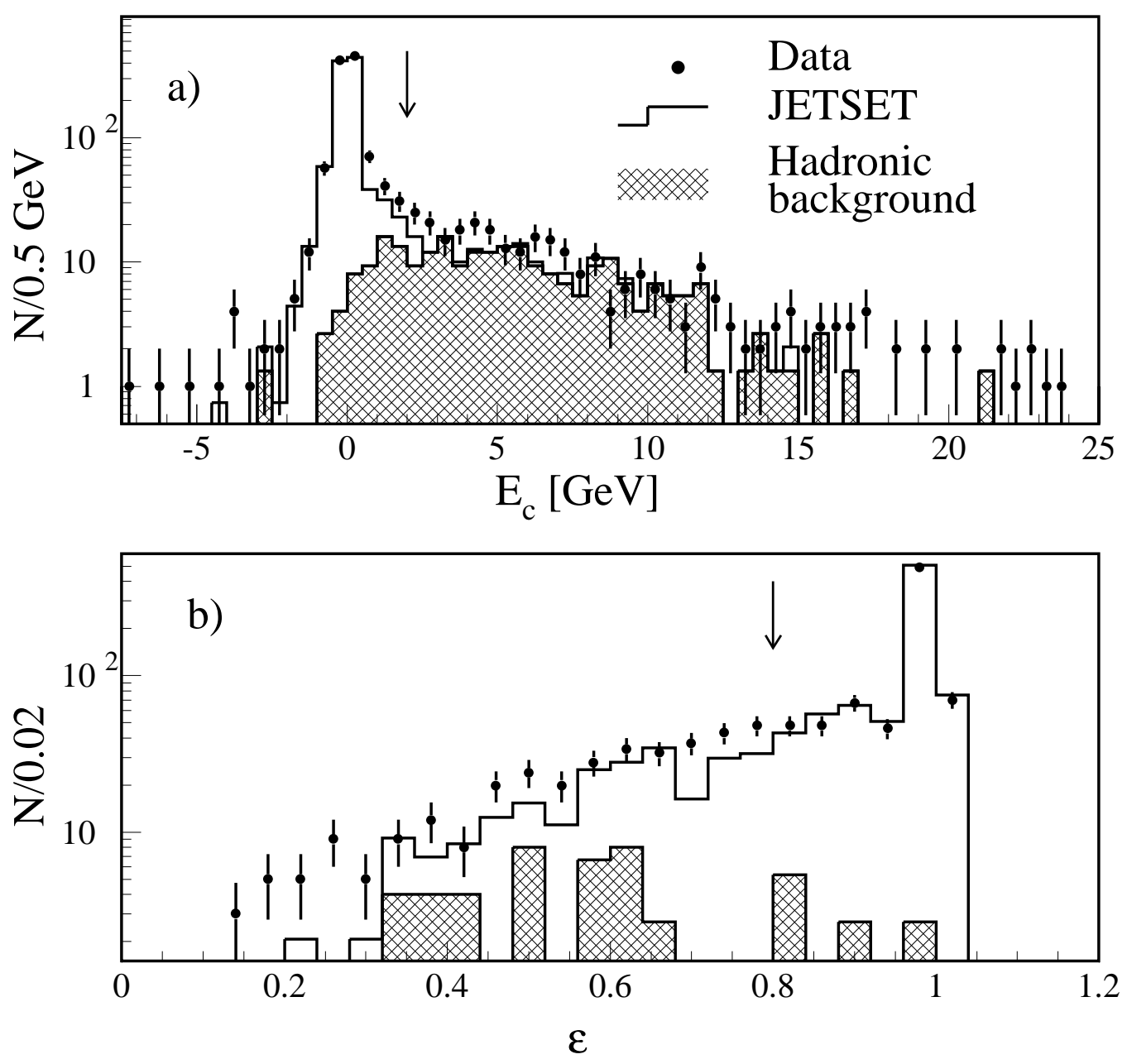

Figure 1: Isolation variables (a) $E_{c}$, (b) $\varepsilon$ after the cut $\left|E_{c}\right|<2 \mathrm{GeV}$ has been applied. Solid points represent the data, while the histogram represents the JETSET prediction. The background contribution from neutral hadrons is shown as the hatched area. The arrows represent the cuts used. 

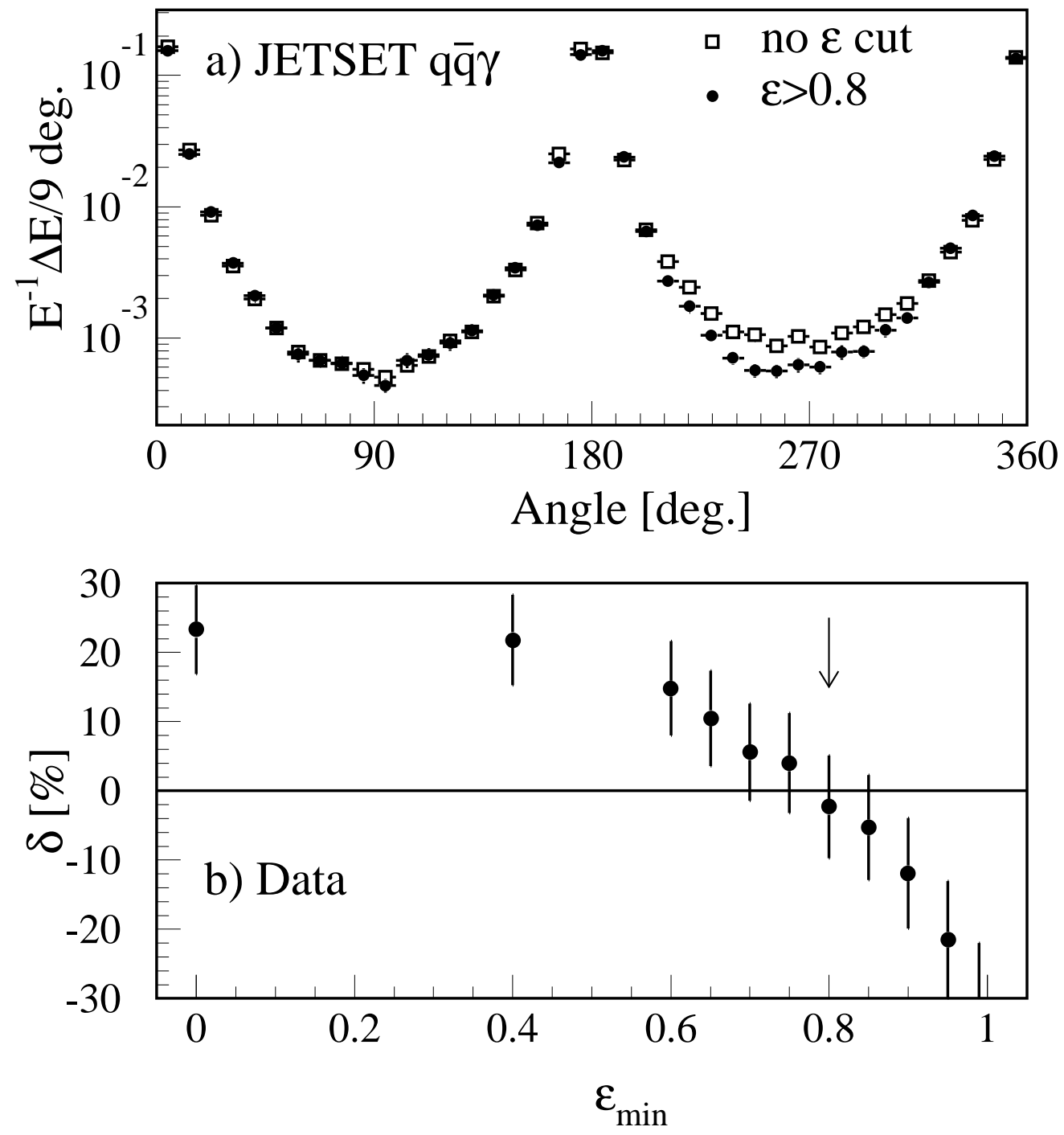

Figure 2: (a) Energy distribution projected onto the event plane in the $q \bar{q}$ rest frame for JETSET $q \bar{q} \gamma$ events after removal of the photon, which otherwise appears around $260^{\circ}$. Angles run from highest energetic jet direction towards the second jet. Neutral hadron background is removed and the $E_{c}$ cut has been applied. (b) Relative energy flow difference $\delta$ between the photon region $\left[234^{\circ}, 297^{\circ}\right]$ and the symmetric one $\left[63^{\circ}, 126^{\circ}\right]$ for data as a function of the cut on $\varepsilon$. 

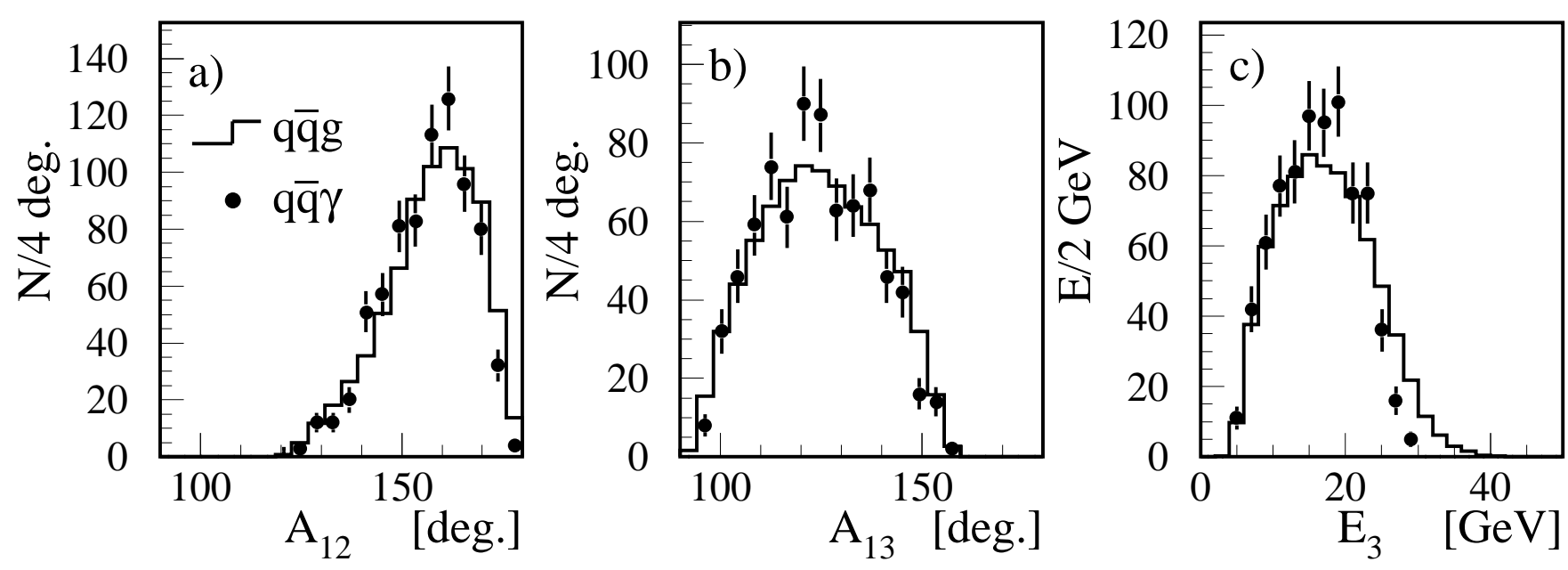

Figure 3: (a) Angle $A_{12}$ between the two quark jets and (b) between the most energetic jet and the third jet $A_{13}$. (c) Energy of the third jet $E_{3}$. 


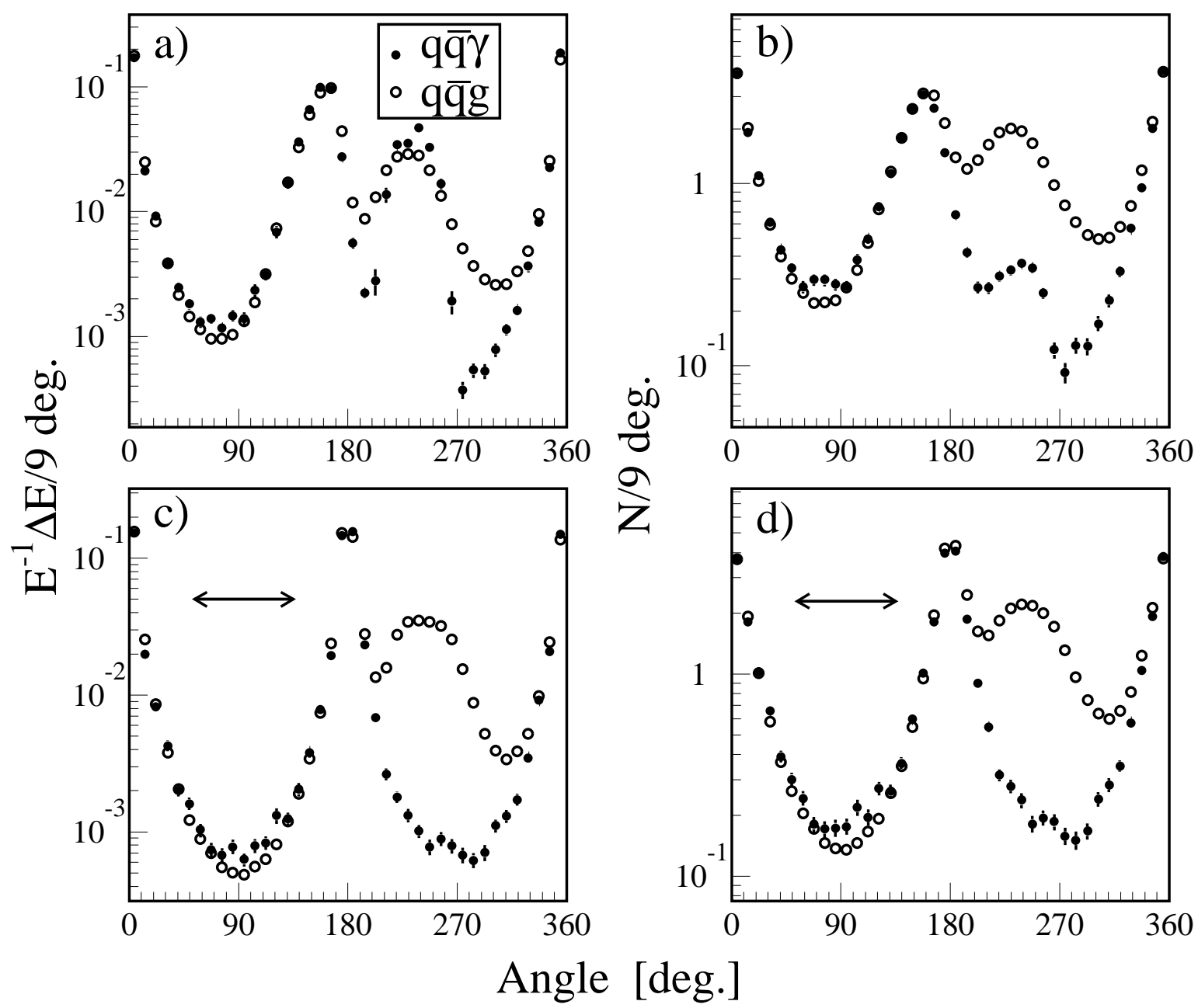

Figure 4: (a) Distribution of the normalized energy flow and (b) particle flow in the laboratory frame. (c) and (d) are the corresponding distributions in the $q \bar{q}$ center of mass frame, after the photon has been removed. The arrows show the angular range used to measure the effect. 


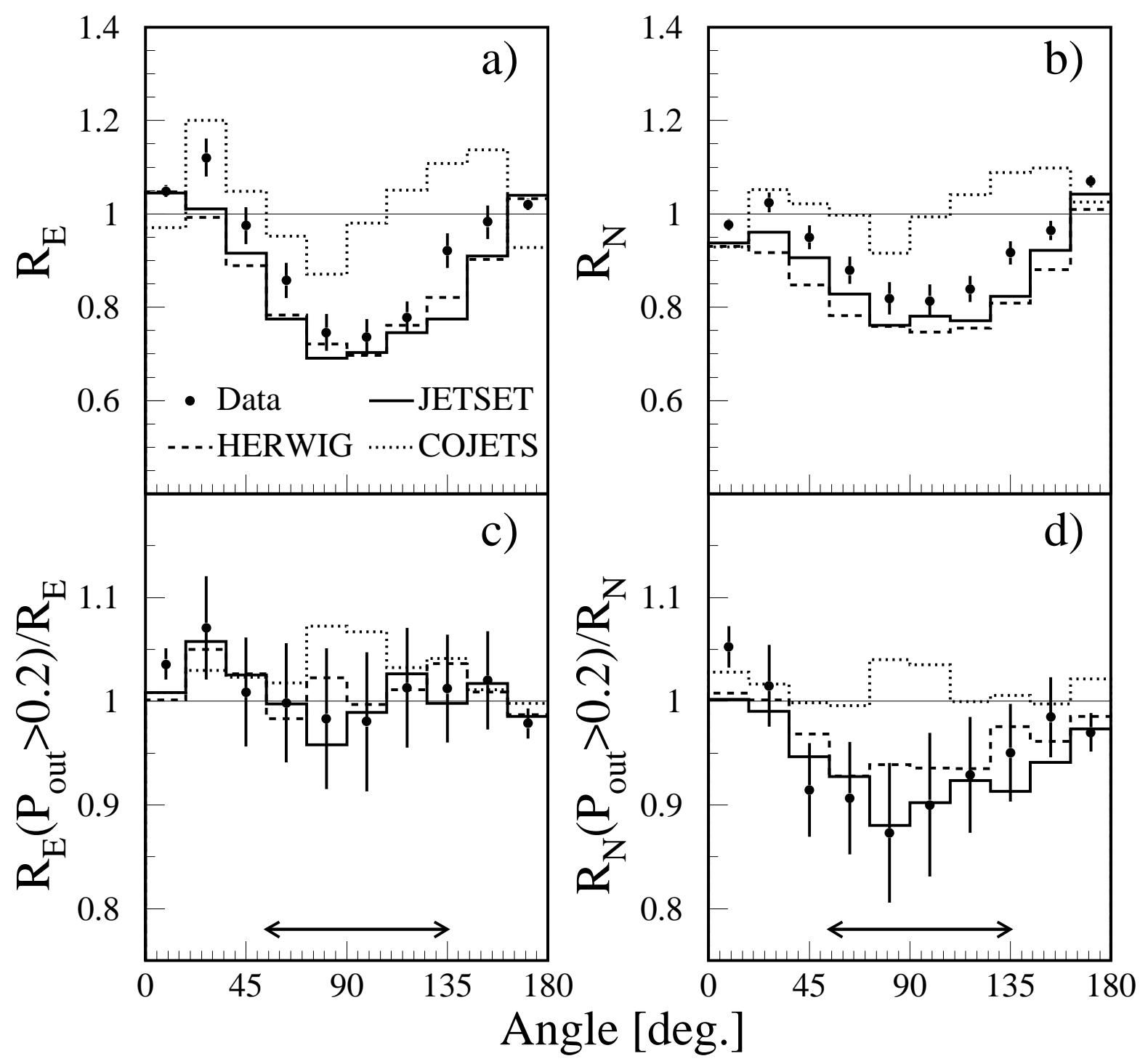

Figure 5: Bin-by-bin ratios of the $q \bar{q} g$ and $q \bar{q} \gamma(a)$ energy and (b) particle flow plots after the application of the algorithm described in the text to $q \bar{q} \gamma$ events. The theoretical predictions have statistical uncertainties of similar magnitude to the ones shown for data. (c) and (d) show the ratios of the distributions with and without a $0.2 \mathrm{GeV} P_{\text {out }}$ cut. Systematic errors are not shown in (a) and (b), while they are negligible in (c) and (d). The arrows show the angular range used to measure the effect. 